\title{
Band-like Stress Fiber Propagation in a Continuum and Implications for Myosin Contractile Stresses
}

\author{
Preethi L. Chandran, Christopher B. Wolf, and Mohammad R. K. Mofrad \\ Molecular Cell Biomechanics Laboratory, Department of Bioengineering, University of California Berkeley, 208A Stanley Hall, \\ Berkeley, CA 94720-1762, USA
}

(Received 25 January 2009; accepted 2 February 2009; published online 11 February 2009)

\begin{abstract}
Stress fibers are band-like features that form with sarcomere-like actin and myosin arrangement between cell regions, resisting myosin contractility. We consider three aspects of stress fiber formation: (1) they form by cytoskeletal actin-myosin interaction when myosin contractile forces are resisted, (2) they propagate in a band-like manner, and (3) they maintain a level of stress and material continuity with the cytoskeleton. This suggests that any description of myosin force should capture the band-like propagation of stress fibers within the constraints of a continuum model. Recent studies describe myosin force as increasing proportional to the cytoskeletal resistance in that direction, but do not capture the band-like propagation of myosin stresses in a continuum. While the spreading of myosin stresses in continuum models is commonly attributed to the elliptic nature of continuum equations, we show that it comes from an incomplete description of the myosin force. Qualitative observations of cytoskeletal actin-myosin interaction indicate the interaction to be 'zipper-like'; myosin contractile forces get transmitted by bending actin filaments in directions away from that of the cytoskeletal resistance. A simple coarse-grained implementation of the lateral myosin forces that arise from the zippering action reproduces band-like stress propagation within a continuum model for the first time. This model also shows actin packing into the stress channel and its propagation along the edge for square and triangular constrained cells; features not captured earlier. Physically, the lateral contractile forces prevent stress spreading by balancing perpendicular shear forces that arise when stress channels through a continuum. Mathematically, these forces render the continuum stress equation hyperbolic. This paper presents a theoretical argument, based on continuum mechanics principles, that it is the zippering actin-myosin action that allows for band-like stress fiber propagation within a coarse-grained cytoskeletal continuum, and that any visualization of the cytoskeletal stress field should account for lateral contractile forces accompanying the much-acknowledged contractile force along a stress fiber.
\end{abstract}

Keywords-Stress fibers, Cytoskeleton, Actomyosin contractility, Coarse-grained cytoskeletal modeling, Zipper-like

Address correspondence to Mohammad R. K. Mofrad, Molecular Cell Biomechanics Laboratory, Department of Bioengineering, University of California Berkeley, 208A Stanley Hall, Berkeley, CA 94720-1762, USA. Electronic mail: mofrad@berkeley.edu actin-myosin interaction, Stress transmission, Lateral contractile forces.

\section{INTRODUCTION}

\section{Background}

Early in the 1970s, prominent bundles of actin filaments were identified in the cytoplasm of stationary nonmuscle cells in culture and in endothelial cells under shear stress. ${ }^{6,24,50}$ Called stress fibers, the actin bundles were found to be contractile $e^{7,36,41}$ and housing myosin filaments, ${ }^{22,81}$ characteristics reminiscent of muscle sarcomeres. Subsequent studies showed that the macromolecular organization of stress fibers was similar to that of sarcomeres in many ways - the actin filaments were in parallel arrangement with filament polarity alternating alongside ${ }^{3,67}$; a lateral spacing of $8-12 \mathrm{~nm}$ between the actin filaments allowed for parallel, bipolar myosin filaments to situate in-between ${ }^{72}$; and the myosin head moved toward the positive pole of the actin filaments during a power-stroke, ${ }^{77}$ producing a net contraction because of the alternating polarity arrangement. The myosin in stress-fibers, myosin II, was found to be a genetic isoform of the myosin II in sarcomeres. ${ }^{54}$ While the two myosins differed in details of filament assembly, regulation and stroke size ${ }^{20}$ they were still found to be functionally interchangeable. ${ }^{45,58}$ Also, a similar group of proteins was found to control the arrangement and interaction of actin and myosin in both stress fibers and sarcomeres. Examples of these proteins are $\alpha$-actinin, filamin, troponin, caldesmon and tropomyosin. ${ }^{27,43,48,49,68,82}$ On the other hand, there are some key differences between stress fibers and sarcomeres. Notably, stress fibers do not bear the strict order and periodicity typical of muscle sarcomeres. ${ }^{60,62,66}$ For instance, myosin and $\alpha$-actinin are not uniformly distributed along a stress fiber; the myosin concentration is greater toward the periphery of a stress fiber, whereas the $\alpha$-actinin concentration is greater in the central 
regions. ${ }^{46,62}$ As a result, the contraction of a stress fiber is not uniform; there is shortening in the peripheral region of the stress fiber, accompanied by simultaneous stretching in the central region. ${ }^{62}$ Therefore, while the many similarities between stress fibers and sarcomeres reflect a similar mechanism of actin-myosin contractility for the two, their differences strongly suggest that stress-fibers are not pre-determined structures like sarcomeres. Rather, the stress fibers arise by rearrangement of the disordered actin cytoskeleton under the contractile action of myosin, and they evolve continually as the constraints on myosin contractility change with cell migration and external force.

Stress fibers form by cytoskeletal actin-myosin interaction when cytoskeletal contractility is resisted. For instance, stress fibers are more pronounced in rigid substrates than soft substrates. ${ }^{29,59}$ They typically form between strong substrate attachments called focal adhesions at which myosin contractility is resisted. ${ }^{7-9}$ The fibers also form along the direction of the external forces and substrate stretch, which would be the direction of cytoskeletal resistance. ${ }^{25,31,44,75}$

Stress fibers propagate through the cell in a bandlike structure. The ability of a cell to form a stress fiber that focuses myosin contractility along a direction of resistance has many functional implications. It allows the cell to stiffen itself in a particular direction to protect against excess stress and strains in that direction. It also allows the cell to scout the matrix rigidity and migrate in the direction of increasing rigidity. ${ }^{53,65}$ Changes in stress fiber patterns, be it due to external forces or strain, lead to changes in cell shape and orientation. $^{21,47,61}$ Also, the band-like propagation of myosin contractility allows the cell to transmit forces across large distances within itself, precisely and without dissipation. ${ }^{5,13,33,57}$

The band-like nature and the systematic recruitment of proteins for stress fiber assembly, $98,42,60,64$ may suggest that stress fibers are discrete structures, independent of the surrounding actin cytoskeleton. However, a number of experimental observations indicate that a level of material and stress continuity exists between the stress fibers and the surrounding actin cytoskeleton. For instance, images of cytoplasmic flow in keratocytes show cytoskeletal actin being compressed into the stress fiber. ${ }^{69,73}$ Hirata et al. ${ }^{31}$ showed that quantum-dot labeled actin filaments in cell lamella follow a definite centripetal trajectory into stress fibers. The directed nature of the trajectory cannot be explained by random diffusion, but only by some coarse-grained material continuity. Wang ${ }^{79}$ showed that as a cell migrates and continually changes its contacts with the matrix, the existing stress fibers realign into the direction of migration. This is possible only if the forces within the stress fibers were to some extent continuous with the stresses in the rest of the cytoskeletal matrix. Note that by stresses within the stress fibers and cytoskeletal matrix, we refer to the contractile stresses of myosin balanced by the tensile resistance of the actin network.

In this paper we attempt to reconcile three aspects of stress fibers: (1) stress fibers form by cytoskeletal actin-myosin interaction when myosin contractility is resisted, (2) they propagate in a band-like manner, and (3) they maintain a level of stress and material continuity with the cytoskeletal matrix. The third aspect suggests that, at a coarse-grained level, the principles of continuum mechanics should govern the stress and strain transfers involved in stress fiber formation. Therefore, combining the three aspects, we require that a correct description of cytoskeletal myosin contractility should capture the band-like propagation of stress fibers within the constraints of a continuum model.

To our knowledge, there is only one continuum model of cell contractility that attempts to capture the formation of stress fibers and that describes the myosin contractile force as increasing proportional to the cytoskeletal resistance. ${ }^{18,19}$ This description of myosin contractile force is intuitive and is validated by experimental observations which show the stiffness of a cell increasing proportional with the stiffness of the resisting substrate. ${ }^{70}$ It is also a version of the Hill's equation of muscle contractility, extended to describe cytoskeletal myosin contractility. However this model does not capture the band-like propagation of stress fibers. ${ }^{18}$ Also it shows stress fibers spreading along diagonals in cells constrained at the corners of a square and triangle geometry. ${ }^{18}$ Experimental observations indicate otherwise: they show the stress fibers forming along the edges in cells under comparable constraints. ${ }^{39,51,74}$

The central focus of this paper is to explore, from a continuum mechanics viewpoint, the nature of cytoskeletal actin-myosin interaction and myosin contractility that could lead to band-like stress fiber propagations in cells. We show that the description of myosin contractility in Deshpande et al. ${ }^{18,19}$ omits a component of the myosin contractile force that is lateral or perpendicular to the direction of cytoskeletal resistance. In the Methods and Results section we show that a simple mathematical implementation of the lateral contractile force produces band-like stress fiber propagation in a continuum. But first, in the rest of the Introduction section, we lay out our argument for the existence of the lateral contractile forces in the following way. We describe some of the current continuum models of cell contractility and detail the features of stress fibers not captured by these models. We then examine the problem of band-like stress propagation from a continuum mechanics viewpoint. 
A common argument made against band-like stress propagation within a continuum is that the straincompatibility requirement makes the continuum stress equations elliptic, and the solutions of elliptic equations tend to spread. We use a different reasoning. We attribute the spreading to the more basic requirement of balancing lone moments that arise with channeled stress propagation. Therefore an appropriate description of myosin contractile stress that balances these moments may still produce band-like propagation. Qualitative experimental observations of actin-myosin interaction in disordered cytoskeleton indicate that it can be described by as zippering, whereby contractile force from an oriented myosin filament is transmitted to directions away from it by bending actin filaments. The zippering action implies that a component of the myosin contractile force is lateral or perpendicular to the direction of cytoskeletal resistance.

\section{Stress Fiber Patterns from Continuum Models}

Myosin contractility has always been an essential feature of continuum models describing cell migration. Alt and Dembo ${ }^{1}$ and Dembo and Harlow ${ }^{16}$ described cytoskeletal contractility as an isotropic inward pressure that opposes the outward hydrostatic pressure of the cell, and increases as a function of the network volume fraction. Gracheva and Othmer ${ }^{28}$ described cytoskeletal contractility as an isotropic active stress that is a function of myosin accumulation due to myosin binding and activation kinetics, and to biochemical signaling. Neither of these models accounts for the increase in myosin force and its accumulation into stress fibers as a function of cytoskeletal resistance. On the other hand, significant progress has been made in the study of the dynamics of a single contractile bundle or stress fiber ${ }^{2,4,11,14}$ and in modeling actin-myosin interaction in solution. ${ }^{37,52,55}$ The cell contractility model of Deshpande et al. ${ }^{18,19}$ addresses the accumulation of myosin contractility and stress fibers as a function of cytoskeletal resistance. The underlying idea in the model can be described as follows: A general cytoskeletal contractility pervades the cell. The resistance of the actin cytoskeleton to myosin contractility varies in magnitude and direction at different regions of the cell, due to the constraints placed by substrate attachments and external forces. The myosin contractile stress at a point increases as a function of the cytoskeletal resistance to contractility at that point.

First, the idea that myosin contractile stress increases with resistance of the actin network is a converse of the well-known Hill's equation of muscle mechanics. ${ }^{18,23}$ The Hill's equation states that the velocity of muscle contraction decreases as the load on the muscle increases. In other words, increasing the load that myosin contractile force has to overcome leads to smaller contraction velocities. ${ }^{23}$ The Hill's equation captures the microstructural phenomena where a myosin head which is unable to overcome the applied load remains stalled on the actin filament until more myosins bind, so that the concerted power-stroke can overcome the applied load. The overall contraction velocity decreases because of the time delay in accumulating sufficient myosin binding. Hill's equation captures two critical features of actin-myosin interaction: an increased resistance to myosin contractility leads a decrease in the myosin disassociation constant and to an increase in the number of myosins binding. The Hill's equation was originally formulated for the well-ordered, unidirectional arrangement of actin and myosin in muscle sarcomeres. When carried over to the more disordered cytoskeletal arrangement, the Hill's equation still explains why increased resistance to contractility causes increased myosin binding and stress fiber formation along the direction of resistance. $^{12}$

While the model by Deshpande et al. ${ }^{18}$ predicts the increased accumulation of myosin at the periphery of stress fibers, it fails to capture the following features of stress fibers:

(1) the band-like nature of contractile stress in stress fibers;

(2) the accumulation of actin material into a stress fiber;

(3) the formation of stress fiber along edges in cells attached at the corners of square and triangular geometries.

The model shows the myosin contractile force spreading outward from the matrix attachments like diffusion fronts. ${ }^{18}$ The spreading of contractile stresses implies that they dissipate away from the matrix attachments and have no directed propagation. In addition, no packing of actin filaments into the stress fibers can be inferred from the model results. Finally, the model predicts stress fibers to form along the diagonals in cells contracting under constraints located at the corners of square and triangular geometries. On the other hand, actin-stained images of cells contracting under micro-patterned constraints or micro-needles placed at the corners of square and triangular geometries show distinct actin bundles or stress fibers forming at the edges of the geometry instead of diagonal. ${ }^{39,51,74}$

\section{Stress Spreading and the Strain Compatibility Equation}

The results of the above model can be attributed to its continuum underpinnings. The spreading of contractile stresses along diagonals is also shown by simple 
contractile continuum materials and is commonly attributed to the strain-compatibility condition used for closing the continuum stress balance.

In a two-dimensional continuum stress field, there are four unknown stress components, $\sigma_{i j}$, but only three equations to solve them: the two force balance equations (Eq. 1) and the moment balance equation (Eq. 2).

$$
\begin{gathered}
\frac{d}{d x_{j}} \sigma_{i j}=f_{i} \\
\sigma_{i j}=\sigma_{j i}
\end{gathered}
$$

where $f$ is the external force, and the indices $i$ and $j$ indicate $x$ - and $y$-directions. Consider a passive material for now. Its constitutive equations, relating its deformation stress and strain, add three new equations, but also three new unknowns, the strain components, $\varepsilon_{i j}$. For simplicity sake, consider the material to be isotropic, linear-elastic material, in small-strain deformation, and with zero Poisson ratio and elastic modulus, $E$. Its constitutive equations are

$$
\sigma_{x x}=\frac{1}{E} \varepsilon_{x x}, \quad \sigma_{y y}=\frac{1}{E} \varepsilon_{y y}, \quad \sigma_{x y}=\frac{2}{E} \varepsilon_{x y}
$$

To close the problem formulation, a constraint is imposed on the strain field to ensure that the material is a continuum (without internal fractures and discontinuities). This is done in two ways. One way is to express the three strain components as a function of the matrix displacements, and solve for the displacements instead. This adds two new unknowns ( $x$ - and $y$-displacements, $u$ and $v$ ) but three new straindisplacement equations,

$$
\varepsilon_{y y}=\frac{\partial v}{\partial y}, \varepsilon_{x x}=\frac{\partial u}{\partial x}, \varepsilon_{x y}=.5 *\left(\frac{\partial u}{\partial y}+\frac{\partial v}{\partial x}\right), \varepsilon_{x y}=\varepsilon_{y x}
$$

The alternative way is to pose a strain compatibility condition that defines the relation between strain components in a continuum,

$$
\frac{\partial^{2} \varepsilon_{x x}}{\partial x^{2}}+\frac{\partial^{2} \varepsilon_{y y}}{\partial y^{2}}-2 \frac{\partial^{2} \varepsilon_{x y}}{\partial y \partial x}=0
$$

The compatibility condition is derived by requiring the displacement field to be conservative. ${ }^{56}$ Also, the compatibility condition is but a restatement of the strain-displacement equations (Eq. 4); the strain-displacement equations can be doubly differentiated and added to give back the compatibility condition. ${ }^{56}$ In all, Eq. (5) dictates the nature of the strain field in a continuous body.

Combining the stress-balance equations Eqs. (1)-(3) and the compatibility condition Eq. (5) yields the following equation as governing the stress field in a continuum material.

$$
\frac{\partial^{2} \sigma_{x x}}{\partial x^{2}}+\frac{\partial^{2} \sigma_{x x}}{\partial y^{2}}+\frac{\partial^{2} \sigma_{y y}}{\partial x^{2}}+\frac{\partial^{2} \sigma_{y y}}{\partial y^{2}}=f
$$

An equation of the form in Eq. (6) is 'elliptic' because the coefficients of all terms in the left hand side have the same sign. ${ }^{17}$ As discussed in Blumenfeld, ${ }^{5}$ the solutions to elliptic differential equations are known to spread. A typical example of a physical phenomena governed by elliptic equations is steady-state heat diffusion.

To summarize, the compatibility condition makes the continuum stress field elliptic and therefore predisposed to spread. The argument does not change when an active contractile material is considered, with its contractility a function of the cytoskeletal resistance (see Discussion). However in an active material, the stress that is balanced (Eqs. 1,2, and 6) is the total stress which is the sum of the active contractile stress and material deformation stress. Since the active contractile stress and the material deformation stress act in opposite directions, we refer to the total stress as the residual stress, $\sigma_{i j}^{\mathrm{R}}$, which for the cytoskeletal material is given as,

$$
\sigma_{i j}^{\mathrm{R}}=\sigma_{i j}^{\mathrm{M}}-\sigma_{i j}^{\mathrm{N}}
$$

with $\sigma_{i j}^{\mathrm{M}}$ being the myosin contractile stress, and $\sigma_{i j}^{\mathrm{N}}$ being the stress due to the deformation of the cytoskeletal network. The residual stress, $\sigma_{i j}^{\mathrm{R}}$, is but a measure of the cytoskeletal resistance to myosin contraction.

The compatibility requirement on continuum strain is a common argument made against continuum-based models, in favor of discrete ${ }^{35}$ and isostatic models ${ }^{5}$ of cell contractility. Blumenfeld ${ }^{5}$ noted that if the final stress equation (Eq. 6) were to be hyperbolic, i.e., one of the coefficients carried a different sign, ${ }^{17}$ a band-like stress propagation can occur in a continuum. Hyperbolic equations typically govern unsteady convection and wave transmission. To that end, it was proposed that the cytoskeletal network may indeed be isostatic at the discrete network scale. ${ }^{5}$ An isostatic network has its stress-state completely determined by the relative arrangement of its components alone, dispensing with the compatibility conditions. Coarse-graining an isostatic network yielded a hyperbolic differential equation at the continuum level, allowing for stress fiber-like propagation of contractile stresses. However, it is not clear if the cytoskeleton is indeed isostatic, with force balance determined only by the relative arrangement of its components and not by their constitutive properties. Also, an isostatic model does not account for the fact that focused propagation of contractile stress is not an inherent characteristic of the cytoskeletal arrangement, but arises by active rearrangement of the network under myosin forces. 


\section{Stress Spreading and Moment Conservation}

While it may be concluded that the strain compatibility requirement preempts the formation of bandlike stress fibers in a continuum, we take on a different viewpoint. We propose that it is the force- and moment-balance requirements (Eqs. 1 and 2) that predispose stresses to spread in a continuum. We depict our argument pictorially in Fig. 1 with respect to the moment-balance requirement (Eq. 2). Consider the compressive stress field with a rigid block due to a uniform weight placed on it (Fig. 1a). Note that a passive compressive stress resisting a 'pushing' load is equivalent to active contractile stress resisting a "pulling' load. For a rigid block, the strain gradients are zero, and the compatibility condition is trivially satisfied, and the stress field is no longer limited by the strain-compatibility arguments. Now consider the possibility that the compressive stress due to the weight may channel right through the rigid block without spreading (Fig. 1b). We can test if such a field is possible by checking the force and moment balance over all control volumes (CVs) within the rigid block. In Fig. 1b, CV 1 and CV 2 are situated completely outside and completely inside the stress channel, and the net

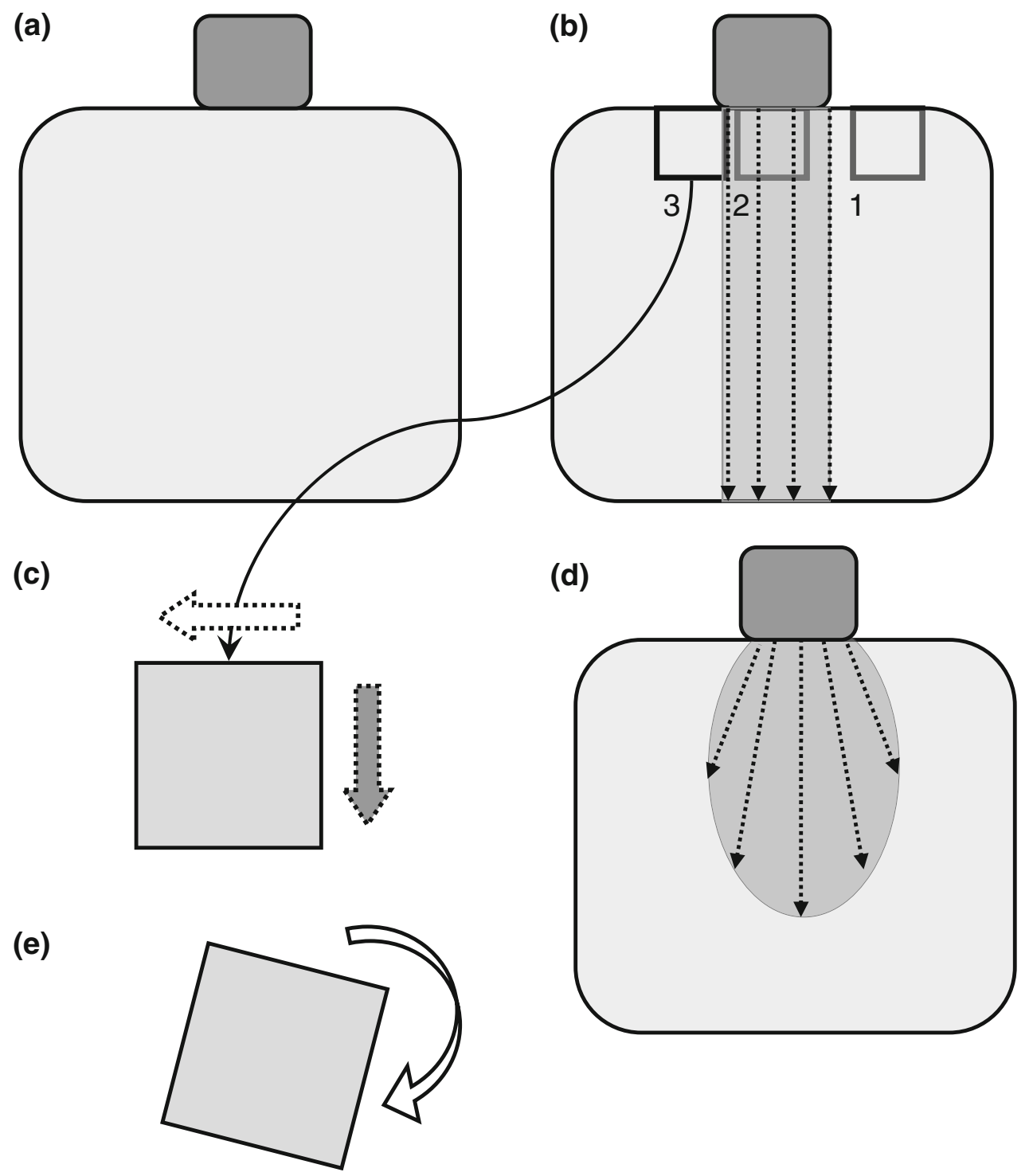

FIGURE 1. An argument for stress spreading. (a) Weight placed on rigid block. (b) Example of a possible channeling stress field within the rigid block due to weight placed on it. For the stress field to exist, the stress conservation equations should hold over all control volumes within the block. Three sample control volumes are shown. (c) Unbalanced shear forces (gray arrow) on the control volume 3 alongside the stress channel. The vertical shear force needs to be balanced by a horizontal shear from the channel (white arrow). (d) Expected stress spreading (gray) to supply the unbalanced force in (c). (e) If the block were deformable, the unbalanced shear force would cause the control volume to rotate clockwise away from the weight. 
force and moment over them are expected to be zero. In the case of $\mathrm{CV} 3$, which is situated alongside the stress channel, there is a lone shear force on the right vertical surface, which would rotate the $\mathrm{CV}$ clockwise outwards if not balanced (Fig. 1c). As per the moment-balance equation (Eq. 2), a horizontal shear force is required to provide the anti-clockwise moment to neutralize the moment of the lone vertical shear force (Fig. 1c). Since this horizontal shear force has to come from within the stress channel, the channel must spread to generate it (Fig. 1d). Also note that if the block were not rigid, the unbalanced vertical shear force would rotate the $\mathrm{CV}$ clockwise outwards and away from the channel as part of block's deformation, causing the compressive strain to spread (Fig. 1e).

Therefore, in a passive continuum material, the requirement for moment balance can be considered a more fundamental reason why stresses spread. This opens the possibility that any active contractile force, capable of providing the perpendicular force to balance the moments of a stress channel, can propagate a band-like stress fiber. To test this we revisit some of the qualitative experimental observations on cytoskeleton actin-myosin interaction.

\section{Kinematics of Cytoskeleton Actin-Myosin Interaction}

While the kinematics of actin-myosin interaction in muscle sarcomeres is well studied, ${ }^{15,34}$ little is known about how the kinematics translates to the disordered actin arrangement of non-muscle cytoskeleton. However a qualitative picture emerges upon examining some experimental observations. Consider the experiment by Suzuki et al. ${ }^{71}$ in which myosin filaments were immobilized on a coverslip in the figure of 8 . The myosin heads were able to pick randomly placed actin filaments from different directions and transport the actin filaments parallel to themselves. ${ }^{71}$ In other words, an immobilized myosin filament was able to bind actin filaments from different directions, and zipper the filaments in to be parallel to itself. A zippering-in action of myosin on actin filaments was also imaged by Schaub et al. ${ }^{69}$ in the retraction fibers of cytochalasintreated keratocytes. A zippering-in action suggests two requirements for actin-myosin binding: (1) that the initial binding is flexible with relaxed constraints on actin-myosin orientation, and (2) that end binding is strict with a preferred parallel actin-myosin orientation. Experimental observations suggest that such is the case. Crystallographic and molecular-dynamics studies show that the myosin head is flexible in the unbound state, undergoes random Brownian motion and has a weak initial binding with actin filaments. ${ }^{15,26,80}$ A preferred parallel end-binding is suggested by the parallel arrangement of actin filaments radiating from actin-myosin clusters in cytochalasin-treated fibroblasts $^{77}$ and by crystallographic/modeling studies which suggest that the tight binding of myosin with actin occurs in only one direction. ${ }^{63}$ Further, SEM images of actin-myosin interaction in keratocyte cytoskeleton show the actin filaments bending to be parallel to each other at the local interaction site, inspite of the overall random orientation of the cytoskeletal actin filaments. $^{73}$

Consider the qualitative kinematics involved in the zippering-in action between a strongly bound myosin filament and a randomly oriented actin filament placed on it. Zippering would involve bending the local portion of the actin filament to be parallel to myosin and sliding it along under the myosin power-stroke (Fig. 2). The force of the myosin power-stroke is transmitted along the actin filament to the rest of the network, and due to the filament bending and orientation, the force is felt in directions not necessarily parallel to the myosin (Fig. 2). In other words, the contractile force of myosin which, according to Hill's equation, is proportional to the network resistance in that direction, is felt along other directions too, as long as there are actin filaments oriented in those directions and the actin filaments have the flexibility to bend. ${ }^{11}$ Therefore, when translating Hill's muscle equation to the disordered cytoskeletal network, the maximal myosin force cannot be considered to be restricted to the direction of maximal resistance.

In a coarse-grained sense, the force due to the zippering action of cytoskeletal actin-myosin can be decomposed into two components: a strong contractile component along the direction of maximum resistance, and a significant contractile component perpendicular to the direction of resistance. We refer to the latter contractile force as lateral forces. Note that by virtue of the zippering action, lateral forces exist only as long as there are actin filaments in the region of the stress fiber that are oriented away from the stress fiber. This is different from the Hill's equation conception, ${ }^{18}$ where lateral forces also exist as long as there are actin filaments oriented away from the stress fiber, but being proportional to the resistance in those directions, the lateral forces diminish rapidly.

While there is no direct way to infer the presence of lateral forces around a stress fiber, indirect evidence can be obtained from some experiments. Schaub et $a l .{ }^{69}$ observed a strong perpendicular motion and packing of actin filaments into the main stress fiber of migrating keratocytes. This suggests the presence of a lateral compressive force around the stress fiber. We also note that Svitkina et $a l^{73}$ and Verkhovsky et al. ${ }^{78}$ implied the myosin force to have a parallel and perpendicular component in their Network Contraction 
(a)

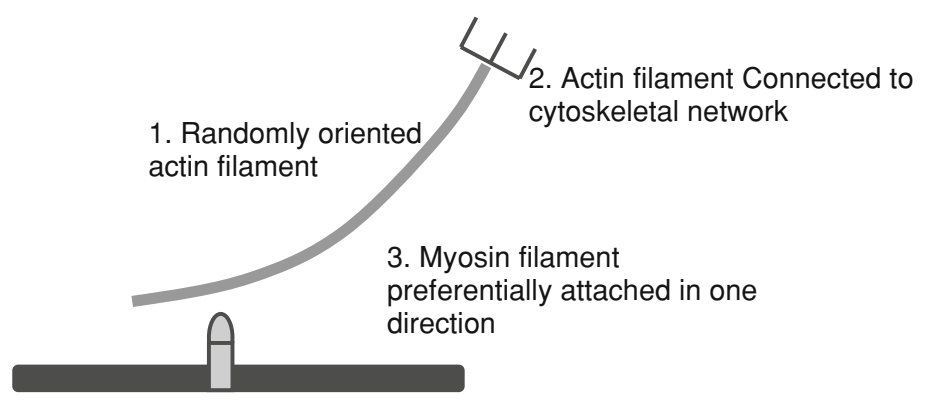

(b)

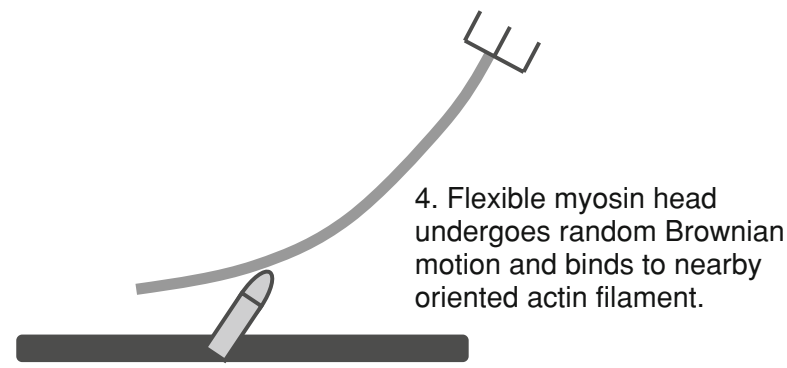

(c) 4. Myosin power-stroke rotates the local
portion of the actin filament parallel to itsel while sliding it along.

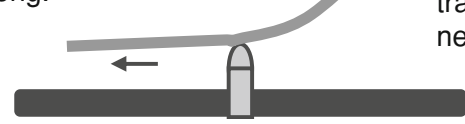

6. Network experiences a net parallel and perpendicular contractile force due to myosin action<smiles></smiles>

5. Actin filament bending to accommodate myosin sliding force and network resistance. Myosin force transmitted along actin filament to network.

FIGURE 2. Proposed sequence of events in the zippering interaction between myosin and disordered actin. The zippering action transmits myosin force through bending actin filaments to directions away from the myosin filament.

Model which describes cytoskeletal rearrangements in migrating keratocytes. Finally, a strong actin cleavage is usually observed around the stress fiber in migrating keratocytes. ${ }^{73}$ The increased flexibility of the actin filaments around stress fibers suggests that, during the formation of stress fibers, there is decreased cytoskeletal resistance to lateral contractile forces.

To summarize, we argued that the description that myosin contractility is proportional to cytoskeletal resistance does not capture the band-like nature of stress fibers because it omits a component of myosin force that is transmitted lateral to the resistance direction by zippering actin-myosin interaction. To show that accounting for lateral contractile forces will capture band-like stress fiber propagation, we implement a simple, minimalistic coarse-grained model of the zippering actin-myosin interaction in a continuum cell.

\section{METHODS}

\section{Coarse-Grained, Minimalistic Model of Myosin Zippering in Cytoskeletal Network}

We first emphasize that our aim is not to propose a general model of stress-fiber formation, but only to show that the band-like propagation of stress fibers can be modeled within a continuum material if an appropriate description of actin-myosin interaction is used. We do not consider other cytoskeletal activity involved in the formation of stress fibers, like actin polymerization, actin crosslinking, biochemical signaling, etc., which though necessary for stress fiber formation, do not affect our qualitative argument. Also, since our aim is not to present a detailed microstructural model of actin-myosin zippering, we only make a coarse-grained approximation of its net effect which could be easily implemented in a continuum stress-balance. 
By the Hill's description of actin-myosin interaction, the maximum myosin force at any point occurs only in the direction of the maximum network resistance there. However, by the zippering action of actinmyosin interaction, the maximum myosin force is transmitted to actin filaments in other directions too. The simplest way to approximate the zippering action is then by requiring that the maximum myosin force at a point acts on all actin filaments at that point, irrespective of orientation. Therefore, if $\sigma_{i j}^{\mathrm{M}}$ is the myosin stress, the increase in myosin stress in a time interval $\Delta t$ is given as,

$$
\Delta \sigma_{i j}^{\mathrm{M}}=K \sigma_{\mathrm{p}}^{\mathrm{R}}\left(C_{0} \sum \Omega_{i j}^{\mathrm{f}} \varepsilon_{i j}^{\mathrm{N}} \Omega_{i j}^{\mathrm{f}}\right)
$$

$K$ is the proportionality constant. $\sigma_{\mathrm{p}}^{\mathrm{R}}$ is the principal maximum value of the residual stress $\sigma_{i j}^{\mathrm{R}}$, and the summation is over all filament orientations.

The description of myosin stress in Eq. 8 is explained as follows. First, Eq. 8 denotes that the myosin force is proportional to $\sigma_{\mathrm{p}}^{\mathrm{R}}$, and therefore proportional to the maximum cytoskeletal resistance, $\sigma_{\mathrm{p}}^{\mathrm{R}}$. Note that the residual stress, $\sigma_{i j}^{\mathrm{R}}$, indicates how much of the myosin stress is resisted by the cytoskeletal network without deforming itself, and is a measure of the cytoskeletal resistance (Eq. 7). Second, our simplest conception of the zippering action is that the same maximum myosin force is transmitted along all actin filaments of the network, irrespective of the filament direction. This can be captured by multiplying the maximum myosin force with the tensor of filament concentrations. The term within the bracket in Eq. 8 gives the filament concentration tensor. It is determined as follows. $\varepsilon^{\mathrm{N}}$ is the average deformation tensor of a network and can be shown be equal to the coarsegrained continuum strain. ${ }^{10} \Omega_{i j}^{\mathrm{f}}$ is the filament orientation tensor, and its components are,

$$
\Omega_{x x}^{\mathrm{f}}=\cos ^{2} \theta^{\mathrm{f}}, \quad \Omega_{x y}^{\mathrm{f}}=\cos \theta^{\mathrm{f}} \sin \theta^{\mathrm{f}}, \quad \Omega_{y y}^{\mathrm{f}}=\sin ^{2} \theta^{\mathrm{f}}
$$

where $\theta^{\mathrm{f}}$ if the filament direction. The term within the summation determines the change in the filament length along a direction as a function of the overall network strain in that direction. The first multiplication of the network strain tensor, $\varepsilon^{\mathrm{N}}$, by $\Omega_{i j}^{\mathrm{f}}$ serves to project it along that orientation. The value of the overall network strain along that direction is taken to determine the change in the total length of filaments (filament 'strain') in that direction. The multiplication by the second $\Omega_{i j}^{\mathrm{f}}$ projects the filament 'strain' in the filament direction, back along the major directions of the stress tensor. Therefore, the overall network strain in each direction is used to determine the change in filament length in that direction. This idea of determining filament length change as a projection of the overall network strain is known as affine transformation, and is commonly used to describe filament kinematics in networks ${ }^{30}$ and fiber composites. The summation in Eq. 8 is over all filament orientations, and $C_{0}$ is the initial concentration of actin filaments in each orientation (or total filament length per unit area in each direction). The term within the bracket is therefore a 'filament strain' tensor which gives the concentration of actin filaments in each direction as a function of the overall strain of the cytoskeletal network, $\varepsilon_{i j}^{\mathrm{N}}$. Note that by considering $C_{0}$ to be constant, the undeformed actin network is assumed to be random.

Let $k$ be some measure of the network stiffness or network resistance to filament rearrangement. Then the increase in network deformation stress in an interval $\Delta t$ due to its deformation and rearrangement of filaments within is given by,

$$
\Delta \sigma_{i j}^{\mathrm{N}}=k\left(C_{0} \sum \Omega_{i j}^{\mathrm{f}} \Delta \varepsilon_{i j}^{\mathrm{N}} \Omega_{i j}^{\mathrm{f}}\right)
$$

where the term within the brackets gives the deformation of the network as a function of the filament rearrangements or the change in filament concentration tensor.

Therefore at each time interval, the residual stress build-up within the cytoskeletal network can be obtained as,

$$
\Delta \sigma_{i j}^{\mathrm{R}}=\Delta \sigma_{i j}^{\mathrm{M}}-\Delta \sigma_{i j}^{\mathrm{N}}
$$

To summarize, the rearrangement of network filaments at a point is determined by the overall network strain at that point. The stiffness of resistance of the network to the filament rearrangements determines the network deformation stress. The myosin contractile stress is determined by the maximum residual stress and acts on all filaments of the network, irrespective of direction. That part of the contractile stress that is not balanced by network deformation is referred to as residual stress, and shows up as tension within the network. The residual stress field should be at equilibrium, and the principle value of the residual stress at a point determines the myosin stress there for the next round of contraction.

The equations were implemented via a user-defined hypoelastic material subroutine in the Marc Mentat Finite Element software (MSC Software Corporation, Santa Ana, CA). The plane-stress version of the continuum stress-balance was solved. Matrix attachments were modeled, in a manner similar to Deshpande et al., ${ }^{18}$ as attachments to foundation springs that exerted an external resistive force $(f$, of Eq. 1 and Eq. 6) but only normal to the cell surface (see Fig. 3 in 


\section{(a)}

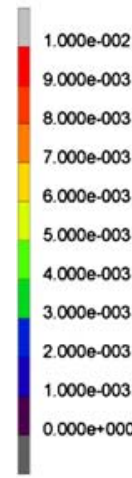

Stress Maxima

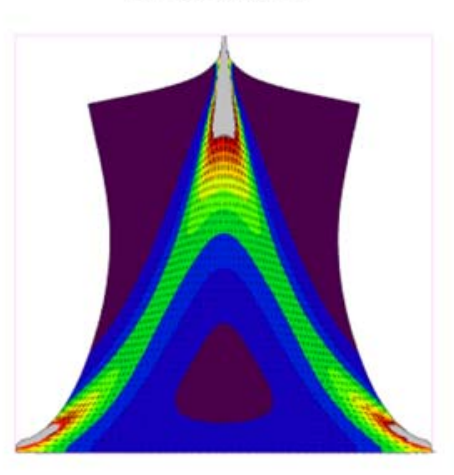

(b)

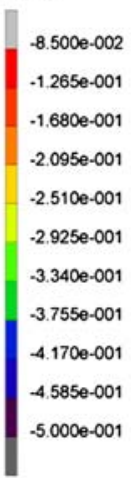

Strain Minima

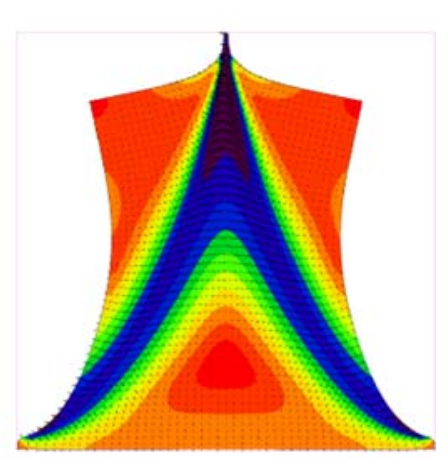

(c)
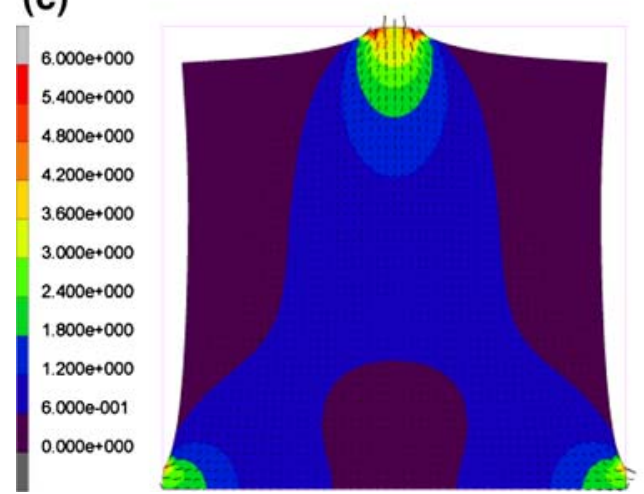

FIGURE 3. Comparing the deformation of a material with actin-myosin like contractility (above) against that of a simple contractile material (below). Contractile material is attached throughout at the bottom surface and at a central region on the top surface. Contractility was simulated using the proposed zippering-like interaction between actin and myosin. (a) The principle maxima of the residual stress due to cell contractility. A clear channeling of stress is seen. (b) The corresponding principle minima of the network strain. The stress channel region is accompanied by a strong lateral compaction, indicating packing of material into stress fibers. (c) The contours of stress maxima for simple contracting material, without the lateral contractile force implied by zippering actin-myosin interaction. A clear spreading of stress is seen.

Deshpande et al. $\left.{ }^{18}\right)$. In the simulations presented, the matrix resistance was assumed to be much greater than the forces of network contraction. The model results are shown in the following section.

\section{RESULTS}

\section{Stress Fiber Patterns in Continuum Model with Zippering Actin-Myosin Interaction}

We first check to see if a zippering action of actinmyosin interaction will produce stress fiber-like propagation of contractile stresses in a continuum medium. We simulate what would be a contractile equivalent of the passive rigid block under compression that was discussed earlier. A square contractile 'cell' is attached to the matrix along its lower horizontal edge, and is constrained at a central region in its opposite horizontal edge. The superposed vector and contour map of the contractile stresses and strains that develop in the cell model after repeated myosin action is presented in Fig. 3. The principal maxima of the residual stress field is shown in Fig. 3a. Two clear channels of contractile stress propagate from the upper constrained region to the corners of the lower attached surface. The principal minima of the continuum strain field is shown Fig. $3 b$. It gives the compressive strains in the actin network. Strong compressive strains occur perpendicular to the stress channel, indicating the lateral packing of material into it (Fig. 3b). Therefore two key characteristics of cell contractility are captured despite the continuum assumptions: (i) the propagation of contractile stress in a band-like manner when there is a resistance to contractility, and (ii), the packing of actin material into the band-like entities called as stress fibers. In Fig. 3c we show the stress map for the corresponding case of a simple contractile material, where the lateral forces of actin-myosin interaction are omitted. A clear spreading of stresses is seen and no lateral packing of filaments into any direction is detected. 
(a)

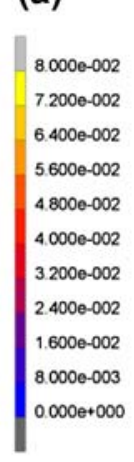

(c)
Residual Stress Maxima
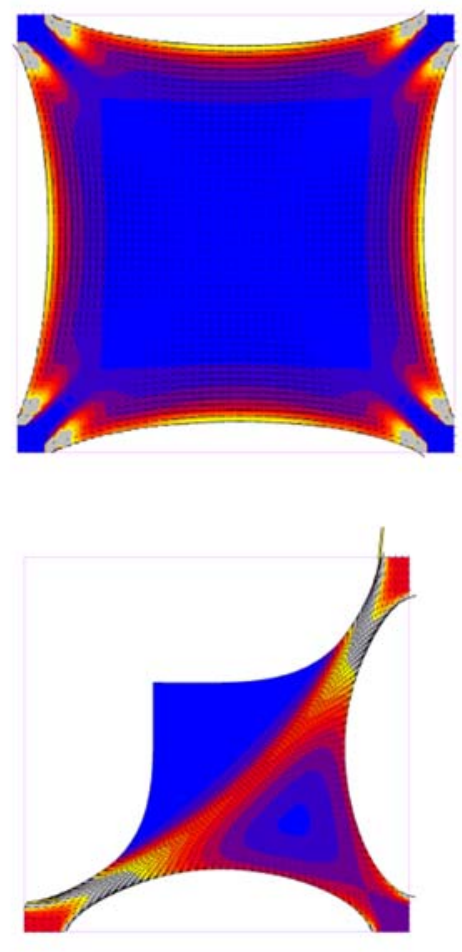

(b)

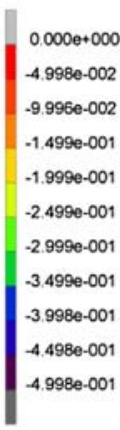

(d)
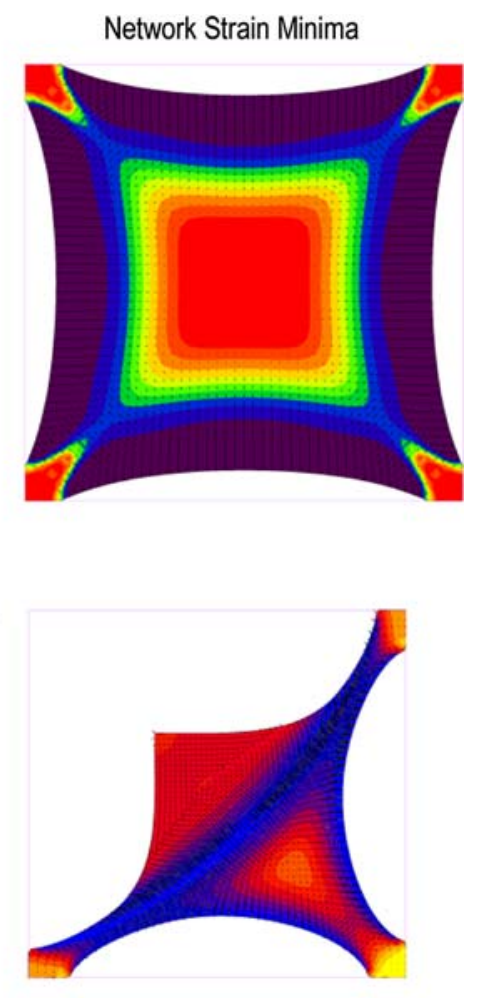

FIGURE 4. Simulating the stress fiber patterns in cells contracting under well-defined micro-patterned constraints. The left panels shows the contours of the principal maxima of the residual stress for a square cell contracting under matrix constraints at the corner of a square (a) and at the corners of a triangle (c). The right panel shows their corresponding principle strain minima (b and d). The channeling of stress maxima and accompanying perpendicular compression indicate that the stress fibers form along the edges of the geometry in both cases.

We then check the patterns of stress fibers formed for cells contracting under constraints at the corner of square and triangular geometries. Previously, we described that continuum models show contractile stresses spreading along the diagonal for these constraints, whereas experiments show stress fibers forming along the edges. The principal maxima of the residual stress field is shown in Figs. 4a and 4c. The residual stresses propagate with definite directionality along the edges of the geometry, and as shown by the map of the principal minimum strain (Figs. $4 \mathrm{~b}$ and $4 \mathrm{~d}$ ), a strong lateral compaction occurs around them. The directed propagation of myosin contractile stresses and the accompanied material packing indicate the formation of stress fibers along the edges of the geometry. Therefore, a simple accounting of the lateral force due to the zippering action of myosin was able to reproduce experimentally observed stress fiber patterns in a continuum setting.

\section{DISCUSSION}

In this paper we tried to reconcile three aspects of stress fiber formation: (1) that they form by cytoskeletal actin-myosin interaction when the myosin contractility is resisted, (2) that they propagate in a band-like manner, and (3) that they maintain a level of stress and strain continuity with the disordered cytoskeletal matrix they arise from. Based on these considerations, we explored the description of actinmyosin contractility that can capture the formation of band-like stress fibers in a continuum setting. An earlier study described myosin contractility as increasing as a function of the cytoskeletal resistance in that direction. However, that description did not capture the band-like stress propagation in a continuum and the patterns of stress fibers seen in cells contracting under simple constraints. While stress spreading in a continuum is traditionally attributed to the strain-compatibility condition which makes the stress equations elliptic and pre-disposed to spread, we suggested that it occurred because of unbalanced moments that came with channeled stress propagation. Therefore an appropriate description of myosin contractile stress can still capture the band-like propagation in a continuum cell.

A qualitative examination of the cytoskeletal actinmyosin interaction suggested it to be of a zippering nature; that myosin undergoes an initial weak binding 
with actin filaments of different relative orientation, but a subsequent power-stroke aligns the actin filament locally into a strong binding. The bending actin filament transmits the myosin force in directions away from the myosin filament. Therefore, the contractile myosin force tends to follow the orientation of the actin filaments than of the myosin filament itself. We refer to the out-of-axis contractile forces as lateral forces. We implemented a simple coarse-grained version of the zippering idea in a continuum model. The model captured the three feature of stress fibers not captured previously: (1) the band-like propagation, (2) the propagation along edges for simple square and triangular constraints, and (3) the compaction of actin filaments into it.

Therefore, by combining continuum analysis and by qualitatively examining cytoskeletal actin-myosin interaction, we proposed in this paper that when stress fiber forms by myosin contraction along the direction of cytoskeletal resistance, a part of myosin contractility occurs perpendicular or lateral to the stress fiber. This perpendicular contractile component serves to bring in actin filaments into the stress fiber and align them, so that the stress fiber can propagate in a band-like manner.

Though we used a simplified minimalistic representation of the cytoskeletal network (homogenous, and isotropic), the kind of stress fiber propagation in our simulation results arises from the description of the myosin forces (as having a force component lateral to the direction of cytoskeletal resistance) and is not dependent on the representation of the cytoskeletal network that was used. Therefore, the ideas presented in this paper can be extended to, and is applicable to, the more complex and realistic descriptions of the cytoskeletal network.

\section{Lateral Forces and Band-like Stress Fiber Propagation in Continuum Models}

We earlier made two continuum mechanics arguments for why stresses spread in a continuum. Within the framework of these arguments, it can be shown why the lateral contractile forces prevent stress spreading.

Consider the case of the compressive stress within a rigid block due to a weight placed on it (Fig. 1). The compressive stress was inferred to spread outwards from under the weight, in order to provide the horizontal force needed to balance the moment from the vertical shear force along the stress channel (Fig. 1). Now, an internal compressive stress due to a weight pushing on the block is similar to an internal contractile stress due to the weight pulling on the block. But if the internal contractile stress is due to actin-myosin zippering action, the vertical contractile forces resisting the weight would be accompanied by strong lateral contractile forces (Fig. 5a). These lateral forces supply the horizontal shear force to balance the moments from the lone vertical shear force, thereby preventing spreading (Fig. 5b). If the block were deformable, a strong lateral contractile force will rotate $\mathrm{CV} 3$ in an counter-clockwise manner toward the stress channel, leading to a strain field that converges a stress channel (Fig. 5c).

We showed earlier that combining the stress balance equations, the constitutive equations and the strain compatibility condition for a simple passive material, yields final stress equations that are elliptic in nature. It can be shown that accounting for the lateral contractile forces will make the final stress equation hyperbolic. Here, we show a simplified derivation that parallels the earlier derivation of the continuum stress equation, Eq. (6). For ease of representation, consider the actin network to be linear elastic and isotropic with zero Poisson's ratio. Note that this is similar to the network description in Eq. (10), were the initial network random and the actin concentration scaled into the network elasticity $E$. The constitutive equations of the material are,

$$
\sigma_{y y}^{\mathrm{N}}=\frac{1}{E} \varepsilon_{y y}^{\mathrm{N}}, \quad \sigma_{x x}^{\mathrm{N}}=\frac{1}{E} \varepsilon_{x x}^{\mathrm{N}}, \quad \sigma_{x y}^{\mathrm{N}}=\frac{2}{E} \varepsilon_{x y}^{\mathrm{N}}
$$

where $\sigma^{\mathrm{N}}$ is the cytoskeletal network stress due to its deformation and $\varepsilon^{\mathrm{N}}$ is the network strain. The network deformation stress is related to the residual stress $\sigma^{\mathrm{R}}$ as,

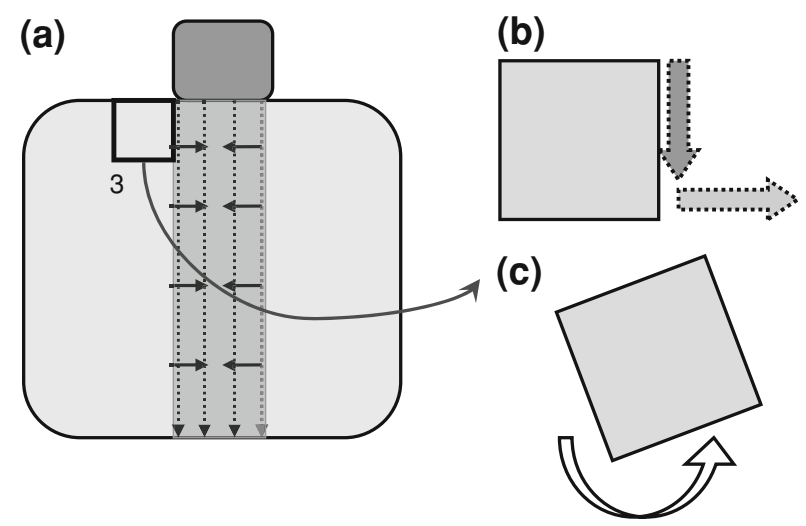

FIGURE 5. Perpendicular contractile force allows for stress channeling. (a) Example of stress channeling with perpendicular contractile force. Control volume 3 of Fig. $1 \mathrm{~b}$ is shown. (b) Unbalanced vertical shear force on control volume can be neutralized by lateral contractile force. (c) Strong lateral contractile force causes clockwise rotation of the control volume inwards toward the stress channel, causing the stress channel to converge. 


$$
\begin{gathered}
\frac{1}{E} \varepsilon_{y y}^{\mathrm{N}}=\sigma_{y y}^{\mathrm{M}}-\sigma_{y y}^{\mathrm{R}} \\
\frac{1}{E} \varepsilon_{x x}^{\mathrm{N}}=\sigma_{x x}^{\mathrm{M}}-\sigma_{x x}^{\mathrm{R}} \\
\frac{1}{E} \varepsilon_{x y}^{\mathrm{N}}=\sigma_{x y}^{\mathrm{R}}
\end{gathered}
$$

Assume that the cytoskeletal network is constrained along the $y$ direction and is free along the $x$ direction. By our description of actin-myosin contractile stress, the vertical component of the myosin stress, $\sigma_{y y}^{\mathrm{M}}$, increases with the residual stress in that direction, $\sigma_{y y}^{\mathrm{R}}$. Let $\varsigma$ be the proportionality constant at any instant. Note that $\varsigma$ can take on any value below 1 . Therefore Eq. (13a) can be rewritten as,

$$
\frac{1}{E} \varepsilon_{y y}^{\mathrm{N}}=(\varsigma-1) \sigma_{y y}^{\mathrm{R}}
$$

Equation (13b) can also be rewritten in a similar form,

$$
\frac{1}{E} \varepsilon_{x x}^{\mathrm{N}}=(\eta-1) \sigma_{x x}^{\mathrm{R}}
$$

where $\eta$ is like a proportionality factor at any instant. However, the cytoskeletal network is free to deform in the $x$-direction, and so $\sigma_{x x}^{\mathrm{R}}$ is small. Therefore a large component of the horizontal myosin stress, $\sigma_{x x}^{\mathrm{M}}$, is contributed by the residual stress in the vertical direction, $\sigma_{y y}^{\mathrm{R}} \cdot \sigma_{x x}^{\mathrm{M}}$ is more likely to reflect $\sigma_{y y}^{\mathrm{R}}$, and since $\sigma_{y y}^{\mathrm{R}} \gg \sigma_{x x}^{\mathrm{R}}, \eta$ is likely to be much greater than $1(\eta \gg 1)$.

Combining the resulting matrix stress-strain equations, Eqs. (14a), (14b), and (13c), with the compatibility condition (Eq. 5) gives

$$
(\eta-1) \frac{\partial^{2} \sigma_{x x}^{\mathrm{R}}}{\partial y^{2}}+(\varsigma-1) \frac{\partial^{2} \sigma_{y y}^{\mathrm{R}}}{\partial x^{2}}=-2 \frac{\partial^{2} \sigma_{x y}^{\mathrm{R}}}{\partial x \partial y}
$$

Also, the stress balance equations (Eqs. 1 and 2) can be differentiated and combined to give,

$$
\frac{\partial^{2} \sigma_{x x}^{\mathrm{R}}}{\partial x^{2}}+\frac{\partial^{2} \sigma_{y y}^{\mathrm{R}}}{\partial y^{2}}=-2 \frac{\partial^{2} \sigma_{x y}^{\mathrm{R}}}{\partial x \partial y}
$$

Combining Eqs. (15) and (16), the stress equation appears as,

$$
\frac{\partial^{2} \sigma_{x x}^{\mathrm{R}}}{\partial x^{2}}+\frac{\partial^{2} \sigma_{y y}^{\mathrm{R}}}{\partial y^{2}}+(1-\eta) \frac{\partial^{2} \sigma_{x x}^{\mathrm{R}}}{\partial y^{2}}+(1-\varsigma) \frac{\partial^{2} \sigma_{y y}^{\mathrm{R}}}{\partial x^{2}}=0
$$

Since $\eta \gg 1$, the third term is negative, making the stress equation hyperbolic and therefore able to handle the propagation of band-like contractile stresses or stress fibers. Note that the strain compatibility condition is still satisfied.
If actin-myosin contractility were governed by the Hill's equation, ${ }^{18,19}$ the myosin stress in the $x$-direction would remain becomes proportional to the residual stress in the $x$-direction. In Eq. (17), this would mean that $\eta<\sim 1$ and $(1-\eta)$ is positive, making the resulting stress equation elliptic. Therefore contractile stresses spread with a Hill's equationlike description of myosin contractility. It can also be shown by a similar argument that stresses spread if the contractile stress were assumed a monotonic function of matrix strain instead. Finally, if the material were passive, $\eta$ and $\varsigma$ go to zero, and Eq. (17) reduces to the elliptic stress equation for a continuum material (Eq. 6).

\section{Peripheral Stress Fibers}

It can be argued that the actin staining along the edges of contracting cells ${ }^{39,51,74}$ is due to the cortical actin network and not stress fibers. However, the existence of edge or peripheral stress fibers in fibroblasts on rigid surfaces have been documented. ${ }^{40,76}$ Katoh et al. ${ }^{40}$ showed that disruption of the peripheral fibers by inhibitors of Myosin Light Chain Kinase resulted in loss of cell shape, whereas disruption of the central stress fibers did not. The researchers also showed that, following the restoration of ATP, peripheral stress fibers contracted first, and that their contraction was near complete before central stress fibers started to contract. These observations suggest that peripheral stress fibers are primary stress fibers which form by actin-myosin contraction and determine the shape of the cell, whereas the central stress fibers are secondary stress fibers which form by actin polymerization in the stress field of the peripheral stress fibers. The insights gained from our study would apply only to stress fibers forming by actin-myosin contraction and not by actin polymerization. A similar case for the two kinds of stress fibers, one forming by actin-myosin contractility and the other by filament polymerization was made by Hotulainen and Lappalainen $^{32}$ in human osteosarcoma cells. The transverse arc stress fiber, formed by lateral fusion of actin myosin bundles, ${ }^{69}$ was disrupted by inhibiting myosin contractility with blebbistatin. ${ }^{32}$ However, the blebbistatin disruption of the dorsal stress fibers, which grow at rates attributable to actin polymerization, was delayed until after the transverse arc stress fibers were disrupted. These observations suggest that the dorsal stress fibers, which form by actin polymerization, are secondary fibers developing in the cytoskeletal stress field due to the transverse arc stress fibers, which form by myosin contraction. 


\section{CONCLUSION}

The aim of this paper was not to formulate a model of stress fiber formation, but to put forth ideas for interpreting the contractile stress field within a cell. The central message is that the presence of a stress fiber indicates both a strong contractile force along a stress fiber and a strong compressive force perpendicular or lateral to it. The lateral forces must be accounted for, if cytoskeletal force transfer needs to be captured within a continuum setting. Understanding the contractile stress field around a stress fiber provides insight into the link between stress fibers and cell shape, the flow of actin within a contractile cell, and the formation and arrangement of secondary stress-fibers in the residualstress field of established stress fibers. The study also shows that the zippering nature of actin-myosin interaction allows for band-like propagation of stress fibers within a coarse-grained cell continuum.

\section{ACKNOWLEDGMENT}

The authors thank Mr. David Kim for his help with the project. Financial support from the National Science Foundation is gratefully acknowledged.

\section{OPEN ACCESS}

This article is distributed under the terms of the Creative Commons Attribution Noncommercial License which permits any noncommercial use, distribution, and reproduction in any medium, provided the original author(s) and source are credited.

\section{REFERENCES}

\footnotetext{
${ }^{1}$ Alt, W., and M. Dembo. Cytoplasm dynamics and cell motion: two-phase flow models. Math. Biosci. 156:207228, 1999.

${ }^{2}$ Bathe, M., C. Heussinger, M. M. A. E. Claessens, A. R. Bausch, and E. Frey. Cytoskeletal bundle mechanics. Biophys. J. 94:2955-2964, 2008.

${ }^{3}$ Begg, D. A., R. Rodewald, and L. I. Rebhun. The visualization of actin filament polarity in thin sections. Evidence for the uniform polarity of membrane-associated filaments. J. Cell Biol. 79:846-852, 1978.

${ }^{4}$ Besser, A., and U. S. Schwarz. Coupling biochemistry and mechanics in cell adhesion: a model for inhomogeneous stress fiber contraction. New J. Phys. 9:425, 2007.

${ }^{5}$ Blumenfeld, R. Isostaticity and controlled force transmission in the cytoskeleton: a model awaiting experimental evidence. Biophys. J. 91:1970-1983, 2006.

${ }^{6}$ Bragina, E., J. Vasiliev, and I. Gelfand. Formation of bundles of microfilaments during spreading of fibroblasts on the substrate. Exp. Cell Res. 97:241-248, 1976.
}

${ }^{7}$ Burridge, K. Are stress fibers contractile? Nature 294:691692, 1981.

${ }^{8}$ Burridge, K., and M. Chrzanowska-Wodnicka. Focal adhesions, contractility and signaling. Annu. Rev. Cell Dev. Biol. 12:463-519, 1996.

${ }^{9}$ Burridge, K., K. Fath, T. Kelly, G. Nuckolls, and C. Turner. Focal adhesions: transmembrane junctions between the extracellular matrix and the cytoskeleton. Annu. Rev. Cell Biol. 4:487-525, 1988.

${ }^{10}$ Chandran, P. L., and V. H. Barocas. Affine versus non-affine fibril kinematics in collagen networks: theoretical studies of network behavior. J. Biomech. Eng. 128:259-270, 2006.

${ }^{11}$ Chandran, P. L., and M. R. K. Mofrad. Rods-on-string idealization captures semiflexible filament dynamics. Phys. Rev. E 79:011906, 2009.

${ }^{12}$ Chen, C. S., J. L. Alonso, E. Ostuni, G. M. Whitesides, and D. E. Ingber. Cell shape provides global control of focal adhesion assembly. Biochem. Biophys. Res. Commun. 307:355-361, 2003.

${ }^{13}$ Chicurel, M. E., C. S. Chen, and D. E. Ingber. Cellular control lies in the balance of forces. Curr. Opin. Cell Biol. 10:232-239, 1998.

${ }^{14}$ Civelekoglu-Scholey, G., A. Wayne Orr, I. Novak, J. J. Meister, M. A. Schwartz, and A. Mogilner. Model of coupled transient changes of Rac, Rho, adhesions and stress fibers alignment in endothelial cells responding to shear stress. J. Theor. Biol. 232:569-585, 2005.

${ }^{15}$ Cooke, R. The sliding filament model: 1972-2004. J. Gen. Physiol. 123:643-656, 2004.

${ }^{16}$ Dembo, M., and F. Harlow. Cell motion, contractile networks, and the physics of interpenetrating reactive flow. Biophys. J. 50:109-121, 1986.

${ }^{17}$ Dennery, P., and A. Kryzwicki. Mathematics for Physicists: Harper's Physics Series. New York, Evanston, London: Harper and Row, 1967.

${ }^{18}$ Deshpande, V. S., R. M. McMeeking, and A. G. Evans. A bio-chemo-mechanical model for cell contractility. PNAS 103:14015-14020, 2006.

${ }^{19}$ Deshpande, V. S., R. M. McMeeking, and A. G. Evans. A model for the contractility of the cytoskeleton including the effects of stress-fibre formation and dissociation. Proc. R. Soc. Lond. A: Math. Phys. Eng. Sci. 463:787-815, 2007.

${ }^{20}$ Eddinger, T. J., and D. P. Meer. Myosin II isoforms in smooth muscle: heterogeneity and function. Am. J. Physiol. Cell Physiol. 293:C493-C508, 2007.

${ }^{21}$ Flavahan, N. A., S. R. Bailey, W. A. Flavahan, S. Mitra, and S. Flavahan. Imaging remodeling of the actin cytoskeleton in vascular smooth muscle cells after mechanosensitive arteriolar constriction. Am. J. Physiol. Heart Circ. Physiol. 288:H660-H669, 2005.

${ }^{22}$ Fujiwara, K, and T Pollard. Fluorescent antibody localization of myosin in the cytoplasm, cleavage furrow, and mitotic spindle of human cells. J. Cell Biol. 71(3):848-875, 1976.

${ }^{23}$ Fung, Y. C. Biomechanics: Mechanical Properties of Living Tissues. New York, NY: Springer, 1993.

${ }^{24}$ Gabbiani, G., M. Badonnel, and G. Rona. Cytoplasmic contractile apparatus in aortic endothelial cells of hypertensive rats. La. Invest 32:227-234, 1975.

${ }^{25}$ Galbraith, C. G., R. Skalak, and S. Chien. Shear stress induces spatial reorganization of the endothelial cell cytoskeleton. Cell Motil. Cytoskeleton 40:317-330, 1998.

${ }^{26}$ Geeves, M. A. The dynamics of actin and myosin association and the crossbridge model of muscle contraction. Biochem. J. 274:1-14, 1991. 
${ }^{27}$ Golji, J., R. Collins, and M. R. K. Mofrad. Molecular mechanics of the $\alpha$-actinin rod domain. PLoS Comp. Biol., 2009 , in press.

${ }^{28}$ Gracheva, M., and H. Othmer. A continuum model of motility in amoeboid cells. Bull. Math. Biol. 66:167-193, 2004.

${ }^{29}$ Grinnell, F., and C. R. Lamke. Reorganization of hydrated collagen lattices by human skin fibroblasts. J. Cell Biol. 66:51-63, 1984.

${ }^{30}$ Head, D. A., A. J. Levine, and F. C. MacKintosh. Deformation of cross-linked semiflexible polymer networks. Phys. Rev. Lett. 91:1081021-1081024, 2003.

${ }^{31}$ Hirata, H., H. Tatsumi, and M. Sokabe. Tension-dependent formation of stress fibers in fibroblasts: a study using semi-intact cells. JSME Int. J. C: Mech. Syst. Mach. Elements Manuf. 47:962-969, 2004.

${ }^{32}$ Hotulainen, P., and P. Lappalainen. Stress fibers are generated by two distinct actin assembly mechanisms in motile cells. J. Cell Biol. 173:383-394, 2006.

${ }^{33} \mathrm{Hu}$, S., J. Chen, B. Fabry, Y. Numaguchi, A. Gouldstone, D. E. Ingber, J. J. Fredberg, J. P. Butler, and N. Wang. Intracellular stress tomography reveals stress focusing and structural anisotropy in cytoskeleton of living cells. Am. J. Physiol. Cell Physiol. 285:C1082-C1090, 2003.

${ }^{34}$ Huxley, A. F. Mechanics and models of the myosin motor: philosophical transactions. Biol. Sci. 355:433-440, 2000.

${ }^{35}$ Ingber, D. E. Tensegrity I. Cell structure and hierarchical systems biology. J. Cell Sci. 116:1157-1173, 2003.

${ }^{36}$ Isenberg, G., P. Rathke, N. Hülsmann, W. Franke, and K. Wohlfarth-Bottermann. Cytoplasmic actomyosin fibrils in tissue culture cells: direct proof of contractility by visualization of ATP-induced contraction in fibrils isolated by laser micro-beam dissection. Cell Tissue Res. 166:427-443, 1976.

${ }^{37}$ Jülicher, F., K. Kruse, J. Prost, and J. F. Joanny. Active behavior of the cytoskeleton. Phys. Rep. 449:3-28, 2007.

${ }^{38} \mathrm{Kamm}$, R. D., and M. R. Kaazempur-Mofrad. On the molecular basis for mechanotransduction. Mech. Chem. Biosyst. 1:201-210, 2004.

${ }^{39}$ Kandere-Grzybowska, K., C. J. Campbell, G. Mahmud, Y. Komarova, S. Soh, and B. A. Grzybowski. Cell motility on micropatterned treadmills and tracks. Soft Matter 3:672-679, 2007.

${ }^{40}$ Katoh, K., Y. Kano, M. Amano, K. Kaibuchi, and K. Fujiwara. Stress fiber organization regulated by MLCK and Rho-kinase in cultured human fibroblasts. Am. J. Physiol. Cell physiol. 280:C1669-C1679, 2001.

${ }^{41}$ Katoh, K., Y. Kano, M. Masuda, H. Onishi, and K. Fujiwara. Isolation and contraction of the stress fiber. Mol. Biol. Cell 9:1919-1938, 1998.

${ }^{42}$ Katoh, K., M. Masuda, Y. Kano, K. Fujiwara, and Y. Jinguji. Focal adhesion proteins associated with apical stress fibers of human fibroblasts. Cell Motil. Cytoskeleton 31:177-195, 1995.

${ }^{43}$ Kolahi, K. S., and M. R. K. Mofrad. Molecular mechanics of filamin's rod domain. Biophys. J. 94:1075-1083, 2008.

${ }^{44}$ Kolega, J. Effects of mechanical tension on protrusive activity and microfilament and intermediate filament organization in an epidermal epithelium moving in culture. J. Cell Biol. 102:1400-1411, 1986.

${ }^{45}$ Kolega, J., M. A. Nederlof, and D. L. Taylor. Quantitation of cytoskeletal fibers in fluorescence images: stress fiber disassembly accompanies dephosphorylation of the regulatory light chains of myosin II. Bioimaging 1:136-150, 1993.
${ }^{46}$ Kolega, J., and D. L. Taylor. Gradients in the concentration and assembly of myosin II in living fibroblasts during locomotion and fiber transport. Mol. Biol. Cell 4:819-836, 1993.

${ }^{47}$ Kumar, S., I. Z. Maxwell, A. Heisterkamp, T. R. Polte, T. P. Lele, M. Salanga, E. Mazur, and D. E. Ingber. Viscoelastic retraction of single living stress fibers and its impact on cell shape, cytoskeletal organization, and extracellular matrix mechanics. Biophys. J. 90:3762-3773, 2006.

${ }^{48}$ Langanger, G., J. de Mey, M. Moeremans, G. Daneels, M. de Brabander, and J. V. Small. Ultrastructural localization of alpha-actinin and filamin in cultured cells with the immunogold staining (IGS) method. J. Cell Biol. 99:13241334, 1984.

${ }^{49}$ Lazarides, E., and K. Burridge. Alpha-actinin: immunofluorescent localization of a muscle structural protein in nonmuscle cells. Cell Motil. Cytoskeleton 6:289-298, 1975.

${ }^{50}$ Lazarides, E., and K. Weber. Actin antibody: the specific visualization of actin filaments in non-muscle cells. Proc. Natl. Acad. Sci. U.S.A. 71:2268-2272, 1974.

${ }^{51}$ Lehnert, D., B. Wehrle-Haller, C. David, U. Weiland, C. Ballestrem, B. A. Imhof, and M. Bastmeyer. Cell behaviour on micropatterned substrata: limits of extracellular matrix geometry for spreading and adhesion. J. Cell Sci. 117:41-52, 2004.

${ }^{52}$ Liverpool, T. B., and M. C. Marchetti. Rheology of active filament solutions. Phys. Rev. Lett. 97:1-4, 2006.

${ }^{53}$ Lo, C.-M., H.-B. Wang, M. Dembo, and Y.-L. Wang. Cell movement is guided by the rigidity of the substrate. Biophys. J. 79:144-152, 2000.

${ }^{54}$ Lofgren, M., E. Ekblad, I. Morano, and A. Arner. Nonmuscle myosin motor of smooth muscle. J. Gen. Physiol. 121:301-310, 2003.

${ }^{55}$ MacKintosh, F. C., and A. J. Levine. Non-equilibrium mechanics and dynamics of motor-activated gels. Phys. Rev. Lett. 100(1):018104, 2008.

${ }^{56}$ Malvern, L. E. Introduction to the Mechanics of Continuous Medium. Upper Saddle River, NJ: Prentice Hall, Inc., 1969.

${ }^{57}$ Maniotis, A. J., C. S. Chen, and D. E. Ingber. Demonstration of mechanical connections between integrins, cytoskeletal filaments and nucleoplasm that stabilize nuclear structure. Proc. Natl. Acad. Sci. U.S.A. 94:849-854, 1997.

${ }^{58}$ McKenna, N. M., Y. L. Wang, and M. E. Konkel. Formation and movement of myosin-containing structures in living fibroblasts. J. Cell Biol. 109:1163-1172, 1989.

${ }^{59}$ Mochitate, K., P. Pawalek, and F. Grinnell. Stress relaxation of contracted collagen gels: disruption of actin filament bundles, release of cell surface fibronectin, and down-regulation of DNA and protein synthesis. Exp. Cell Res. 193:198-207, 1991.

${ }^{60}$ Naumanen, P., P. Lappalainen, and P. Hotulainen. Mechanisms of actin stress fibre assembly. J. Microsc. 231:446-454, 2008.

${ }^{61}$ Noria, S., F. Xu, S. McCue, M. Jones, A. I. Gotlieb, and B. L. Langille. Assembly and reorientation of stress fibers drives morphological changes to endothelial cells exposed to shear stress. Am. J. Pathol. 164:1211-1223, 2004.

${ }^{62}$ Peterson, L. J., Z. Rajfur, A. S. Maddox, C. D. Freel, Y. Chen, M. Edlund, C. Otey, and K. Burridge. Simultaneous stretching and contraction of stress fibers in vivo. Mol. Biol. Cell 15:3497-3508, 2004.

${ }^{63}$ Rayment, I., H. M. Holden, M. Whittaker, C. B. Yohn, M. Lorenz, K. C. Holmes, and R. A. Milligan. Structure of 
the actin-myosin complex and its implications for muscle contraction. Science 261:58-65, 1993.

${ }^{64}$ Romer, L. H., K. G. Birukov, and J. G. N. Garcia. Focal adhesions: paradigm for a signaling nexus. Circ. Res. 98:606-616, 2006.

${ }^{65}$ Saez, A., M. Ghibaudo, A. Buguin, P. Silberzan, and B. Ladoux. Rigidity-driven growth and migration of epithelial cells on microstructured anisotropic substrates. PNAS 104:8281-8286, 2007.

${ }^{66}$ Sanger, J. M., B. Mittal, M. Pochapin, and J. W. Sanger. Observations of microfilament bundles in living cells microinjected with fluorescently labelled contractile proteins. J. Cell Sci. 5:17-44, 1986.

${ }^{67}$ Sanger, J. M., and J. W. Sanger. Banding and polarity of actin filaments in interphase and cleaving cells. J. Cell Biol. 86:568-575, 1980.

${ }^{68}$ Sanger, J. W., B. Mittal, and J. M. Sanger. Analysis of myofibrillar structure and assembly using fluorescently labeled contractile proteins. J. Cell Biol. 98:825-833, 1984.

${ }^{69}$ Schaub, S., S. Bohnet, V. M. Laurent, J.-J. Meister, and A. B. Verkhovsky. Comparative maps of motion and assembly of filamentous actin and myosin II in migrating cells. Mol. Biol. Cell 18:3723-3732, 2007.

${ }^{70}$ Solon, J., I. Levental, K. Sengupta, P. C. Georges, and P. A. Janmey. Fibroblast adaptation and stiffness matching to soft elastic substrates. Biophys. J. 93:4453-4461, 2007.

${ }^{71}$ Suzuki, H., A. Yamada, K. Oiwa, H. Nakayama, and S. Mashiko. Control of actin moving trajectory by patterned poly(methylmethacrylate) tracks. Biophys. J. 72:1997-2001, 1997.

${ }^{72}$ Svitkina, T. M., I. G. Surguchova, A. B. Verkhovsky, V. I. Gelfand, M. Moeremans, and J. D. Mey. Direct visualization of bipolar myosin filaments in stress fibers of cultured fibroblasts. Cell Motil. Cytoskeleton 12:150-156, 1989.

${ }^{73}$ Svitkina, T. M., A. B. Verkhovsky, K. M. McQuade, and G. G. Borisy. Analysis of the actin-myosin II system in fish epidermal keratocytes: mechanism of cell body translocation. J. Cell Biol. 139:391-415, 1997.

${ }^{74}$ Tan, J. L., J. Tien, D. M. Pirone, D. S. Gray, K. Bhadriraju, and C. S. Chen. From the cover: cells lying on a bed of microneedles: an approach to isolate mechanical force. Proc. Natl. Acad. Sci. U.S.A. 100:1484-1489, 2003.

${ }^{75}$ Thomopoulos, S., G. M. Fomovsky, and J. W. Holmes. The development of structural and mechanical anisotropy in fibroblast populated collagen gels. J. Biomech. Eng. 127:742-750, 2005.

${ }^{76}$ Totsukawa, G., Y. Yamakita, S. Yamashiro, D. J. Hartshorne, Y. Sasaki, and F. Matsumura. Distinct roles of ROCK (rho-kinase) and MLCK in spatial regulation of MLC phosphorylation for assembly of stress fibers and focal adhesions in 3T3 fibroblasts. J. Cell Biol. 150:797806, 2000.

${ }^{77}$ Verkhovsky, A. B., T. M. Svitkina, and G. G. Borisy. Polarity sorting of actin filaments in cytochalasin-treated fibroblasts. J. Cell Sci. 110:1693-1704, 1997.

${ }^{78}$ Verkhovsky, A. B., T. M. Svitkina, and G. G. Borisy. Network contraction model for cell translocation and retrograde flow. Biochem. Soc. Symp. 65:207-222, 1999.

${ }^{79}$ Wang, Y. L. Reorganization of actin filament bundles in living fibroblasts. J. Cell Biol. 99:1478-1485, 1984.

${ }^{80}$ Warshaw, D. M. Lever arms and necks: a common mechanistic theme across the myosin superfamily. J. Muscle Res. Cell Motil. 25:467-474, 2004.

${ }^{81}$ Weber, K., and U. Groeschel-Stewart. Antibody to myosin: the specific visualization of myosin-containing filaments in nonmuscle cells. Proc. Natl. Acad. Sci. U.S.A. 71:45614564, 1974.

${ }^{82}$ Yamakita, Y., S. Yamashiro, and F. Matsumura. Microinjection of nonmuscle and smooth muscle caldesmon into fibroblasts and muscle cells. J. Cell Biol. 111:2487-2498, 1990. 